\title{
LA TECHNOLOGIE DU MIEL (')
}

$I^{3} \mathrm{AR}$

\section{J. LOUVEAUX}

Station de Recherches apicoles, Bures-sur-Ivette.

Le miel est arant tont mue clenrée alimentaire. On a tant entendu parler cie valeur thérapeutique du miel, d'usages diététiques du miel, etc... qu'on finit parfois par oublier (que le miel est un aliment comme le pain on le lait et 'fu'il doit parvenir sur la table du consommateur aussi appétissant, aussi bon, aussi agréable à voir et à déguster que possible. Il doit aussi garder toutes ses qualités diététiques et thérapeutiques, bien entendu.

Il existe pour le miel toute une technologie, conme il en existe une pour le pain, le lait, le beurre. I, but de cette technologie est de nous fournir les normes yu'il convient de respecter pour récolter, préparer et vendre un produit sain, digeste, agréable et de bonne conservation. Ce but est conforme aux intérêts du producteur et du consommateur. Il ne s'agit pas de dénaturer ou de sophistiquer un produit pour le rendre plus attrayant aux yeux du client, ainsi que voudraient le faire croire certains. Il s'agit senlement d'apporter à l'acheteur la garantie de qualité alimentaire qu'il est en chroit de réclamer et, au producteur, la satisfaction de vendre à son juste prix un produit irréprochable.

Pour donner à la technologie d'un produit alimentaire les bases solides dont elle a bessin, il faut tout d'abord étudier avec le plus grand soin les caractères physico-chimiques de ce produit. Le pain que nous consommons à chaque repas, le lait et des centaines d'autres produits, ont fait l'objet d'un nombre consiclérable de travaux scientifiques et techniques dont certains peuvent paraître futiles, tels cenx portant sur la tartinabilité du beurre. C'est pourtant re la facilité avec laquelle l'Américain mosen étale son beurre sur le toast du petit déjeuner que dépend finalement une partie de la consommation. L'importance du problème n'a pas échappé aux producteurs de beurre anéricains 'jui ont le plus grand intérêt à ce que leur produit s'étale facilement sur le pain même lorsqu'il sort du réfrigérateur.

Comment résoudre de tels problèmes (et il en est de plus inattendus), sans une connaissance approfondie de la physicue et de la chimie des produits alimentaires? Les problèmes qu'il faut résondre pour le miel sont du même ordre que pour les autres denrées; c'est pourquoi je donnerai tout d'abord 'quelques indications sur ce que nous savons actuellement des propriétés physiques essentielles du miel, puis des progrès qui ont été faits dans ces clernières années dans la connaissance de sa composition chinnique.

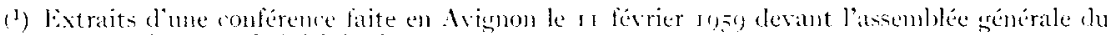
Sumblicat cles P'rodueteurs de Viel de France.

Ambales des droilles. - 105\%. 


\section{Propriétés phỵsiques}

'Toutes les propriétés physiques du miel n'ont pas la même importance en technologie. C'est pourquoi je n'ai retenu pour cet exposé que 5 propriétés essentielles : la densité, 1'indice de réfraction, la chaleur spécifique, la conductibilité thermique et la viscosité. Une bonne connaissance de ces propriétés suffit pour diriger le travail de l'apiculteur dans la plupart des opérations qu'il a à réaliser sur le miel.

Densité. - La densité peut être définie comme le rapport entre le poids de 1'unité de volume d'un corps et le poids du même volume d'eau pure à $4^{\circ} \mathrm{C}$. La densité des liquides peut être connue de façon commode au moyen de densimètres, gradués directement en densités et qui sont des flotteurs de verre équilibrés au moyen de billes de plomb. Avec des liquides très peu visqueux, les densimètres donnent sans délai une indication qui est généralement suffisamment précise pour les besoins courants. Avec le miel il faut prendre beaucoup de précautions pour avoir un résultat précis. Le récipient doit être grand et large ; le miel doit être suffisamment fluide. La lecture ne peut se faire qu'après au moins une heure ou deux de stabilisation. Il y a toujours une certaine imprécision sur la position exacte du flotteur au-dessus du liquide. La densité du miel varie entre $I, 39$ et $I, 44$; on peut assez facilement apprécier le $4^{e}$ chiffre après la virgule; on possède par ailleurs des tables de corrélation entre densité et teneur en eaut qui permettent de connaître avec une bonne approximation la teneur en eau d'un miel, simplement par la lecture de sa densité. Il s'agit là d'une méthode empirique et qui n'a rien d'absolu. Ėn effet, le poids spécifique du miel peut varier légèrement selon l'origine florale et notablement selon la teneur en matières minérales. Pour l'apiculteur qui ne peut pas faire les frais de l'achat d'un réfractomètre la mesure de la teneur en eau des miels par l'intermédiaire de la densité est une méthode tont à fait utilisable.

Indice de réfraction. - Il s'agit d'une propriété optique. 'Tout corps transparent est caractérisé par un certain indice de réfraction. I'indice de réfraction est une constante qui dépend de la nature chimique du corps. Lorsque le corps (n question est en solution dans l'eau, l'indice de réfraction varie régulièrement entre l'indice de l'eau pure et l'indice du corps pur. La mesure de l'indice de réfraction permet donc de connaître facilement la teneur en eau d'un produit en solution tel que le miel. Cette mesure se fait au moyen d'un réfractomètre. Il en existe différents modèles dont certains très peu encombrants tiennent facilement dans la poche et permettent de faire très rapidement des mesures dont la précision est satisfaisante. Une goutte de miel parfaitement liquéfié écrasée entre les prismes de l'appareil suffit pour une mesure.

Les tables de Chataway donnent la correspondance directe entre indice de réfraction et teneur en eau. Ces tables ont fait l'objet de critiques puis de révisions. On peut les considérer comme valables à 0,5 p. Ioo près de la teneur en eau mesurée.

Le problème de la mesure de la teneur en eau des miels est un des plus épineux et des plus controversés. En fait, chaque méthode donne des résultats légèrement différents et il est difficile de choisir une méthode étalon. Pour les besoins courants, les méthodes de densité et d'indice de réfraction sont suffisamment correctes. On peut et on doit les utiliser en raison des services considérables qu'elles rendent en technologie du miel. 
Chaleur spécifique. - Avec la conductibilité thermique la chaleur spécifique est de la plus grande importance pour la résolution des problèmes de chauffage du miel.

La chaleur spécifique d'un corps est la quantité de chaleur nécessaire pottr élever d'un degré la température d'une unité de poids du corps. La petite calorie se définit comme la quantité de chaleur nécessaire pour élever de $I^{0}$ (entre $\mathrm{I}_{4}$ et $\mathrm{I}^{\circ}$ ) la température de $\mathrm{I} \mathrm{g}$ d'eau pure. Par définition la chaleur spécifique de l'eau est de I. Celle du miel n'est, selon Herver (I954), que de 0,54 à $20^{\circ}$ et pour une teneur en eau de $\mathrm{r} 7 \mathrm{p}$. Ioo. Ce qu'il faut noter, et qui est très important, c'est qu'il faut environ deux fois moins de calories pour chautfer du miel que pour chauffer de l'eau.

Conductibilité thermique. - C'est la qualité d'un corps de permettre 1a diffusion de la chaleur dans sa masse. Fille est sujette à des variations considérables entre ce qu'elle est pour les métaux et ce qu'elle est pour des matériaux tels que le liège. On exprime la conductibilité thermique $(\lambda)$ en cal. $\mathrm{cm} . \mathrm{sec} .{ }^{\circ} \mathrm{C}$. Pour l'eau pure, qui n'est pas très bonne conductrice de la chaleur : $\lambda=I_{4}$ $\times 10^{-4}$ à $20^{\circ}$

Pour le miel, $\lambda=\mathrm{I}, 29 \times 10^{-4}$ à $0^{0}$ (pour un miel cristallisé à 20 p. Ioo d'eau) soit près de ro fois plus faible que pour l'eau. La cire est encore plus mauvaise conductrice : $\lambda=0,92 \times 10^{-4}$ à $15^{\circ}$.

Bien entendu la conductibilité thermique varie avec la température et la teneur en eau. On peut dire pratiquement que le miel à 20 p. Ioo d'eau conduit très mal la chaleur à basse température. Il la conduit mieux à chaud et lorsqu'il est très riche en eau, bien que toutefois un miel très déshydraté retrouve brusquement une bonne conductibilité.

Ires conséquences de cette mauvaise conductibilité thermique sont considérables en technologie du miel. Le miel est facile à chauffer puisqu'il a une faible chaleur spécifique; par contre il diffuse très mal la chaleur qu'on lui communique, surtout à l'état solide oì les courants de convexion ne peuvent pas contribuer à équilibrer les températures. Il est donc impossible de réchauffer rapidement et sans surchauffe locale des masses de miel importantes. Par ailleurs, il faut noter que le miel chaud ne reviendra que lentement à la température ordinaire s'il est en masses compactes (1).

Viscosité. - I lans tous les problèmes de pompage, de circulation et de filtration la viscosité intervient comme un facteur essentiel. C'est pourquioi il est du plus haut intérêt de connaître les lois qui régissent cette viscosité.

Il convient d'abord de distinguer deux types de miels du point de vue de la viscosité : les miels normaux, qui suivent les lois de Newton sur la viscosité (miels newtoniens) et les miels anormaux (non-newtoniens) qui présentent du point de vue de la viscosité des anomalies qui peuvent être de plusieutrs ordres ; en général il s'agit de miels thixotropes tels que les miels de Callune.

Pour les miels newtoniens la viscosité peut être reliée d'une façon correcte à la teneur en eau et à la température. Les courbes de viscosité établies par Muxro (I943) sont tout à fait parlantes. On constate facilement à la lecture de ces courbes que la zone sensible se situe entre 30 et $45^{\circ}$. Au-dessous de $30^{\circ}$ la viscosité est généralement trop élevée pour que le miel puisse circuler facile-

(1) Pour ces questions voir l'cutude publice dans les Annales de lableille (Trerer't et Lotveatx, 1958) sur la refonte du miel. 
ment dans les pompes, les canalisations et les filtres. Sa clarification par décantation ne peut pas non plus se faire normalement car les particules solides et les bulles gazeuses rencontrent dans leur déplacement une résistance trop considérable. Les lois de Srockes sur le déplacement des particules solides au sein d'un fluide font en effet intervenir la viscosité à cóté de la différence des densités et du diamètre des particules. Nous reviendrons sur cette question à propos de l'épuration des miels. I'ne température de $35^{\circ}$ parait très suffisante pour assurer à un nuiel normal la fluidité dont il a besoin pour circuler clans les divers appareils de la miellerie.

Il en va tout autrement avec les miels non-newtoniens dont le prototype est le miel de callune. Ces miels sont doués de thixotropie c'est-à-dire que leur viscosité, au lieu d'être fixe, est susceptible de varier dans de larges limites dans le temps, sans que soient modifiées ni la température ni la teneur en ean. En fait, ils sont capables de passer de l'état de gel à l'état de sol sous l'action d'une agitation et de revenir ensuite progressivement à l'état de gel. I,es caractéristiques physiques des miels de Callune ont fait l'objet d'études très poussées de la part de PRYCF-JoNes (I 153 ) qui a mis au point tout un appareillage physique fort compliqué pour mesurer les variations de viscosité des miels thixotropes. Le temps nous manque pour entrer dans le détail de ces expériences. Notons seulement que les miels de Callune ne sont pas vrainent thixotropes au sens que les physiciens attachent à ce mot. I'n effet, il n'y a jamais retour à l'état de sol parfait mais senlement une forte diminution de l'état de zel sous l'influence de l'agitation. Par ailleurs, on sait maintenant que la thixotropie du miel de Callume est due à la présence d'une protéine; cette pro-

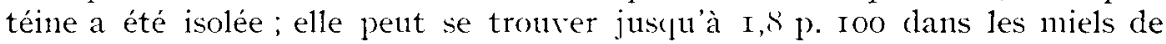
Callune les plus purs qui présentent au plus haut point les propriétés physiques dont il vient d'être question. Le clauffage et le vieillissement naturel ne font ' lu'accentuer les phénomènes de thixotropie. In point de vue technologique les miels de Callune posent des problèmes très ardus mais très intéressants. Letur viscosité anornale interdit évidemment de les traiter comme des miels ordinaires.

Une anomalie moins connue de la riscosité est la dilatance. Les miels donés de cette propriété ont une viscosité qui varie considérablement en fonction de la vitesse d'écoulenlent. Plus cette vitesse est grande plus la viscosité est élevée, si bien cqu'à la vitesse de rotation normale de l'extracteur le miel se présente sous forme de filaments à très haute viscosité qui finissent par blo¡uer l'extracteur. I,es miels dilatants sont riches en dextrines à très haut poids moléculaire. Ce sont principalement des niels d'Eucalyptus.

Connaissant maintenant les propriétés physiques fondamentales du miel, nous sommes déjà mieux armés pour le travailler dans de bonnes conditions. Tous savons mesurer sa teneur en ean et nous savons que sa viscosité, donc la facilité avec laquelle il va circuler, dépend de cette teneur en eau et de la température. Par ailleurs, nous savons que le miel est relativement facile à échauffer mais qu'il conduit très mal la chaleur. Si le miel était un corps simple de la chimie, aussi nettement cléfini que le sucre ordinaire, nous pourrions nous arrêter là. Mais le miel est un produit extrêmement complexe ; c'est un produit biologique presque aussi compliqué que le lait. Il est donc très important de connaitre sa composition de façon précise, ne serait-ce que pour savoir quelles seront les conséquences des opérations technolngiques auxquelles nous nous livrons.

Voyons donc ce cue l'on sait de la composition des miels ou plus exacte- 
ment quels progrès on a fait dans ces dernières années dans la connaissance de cette composition car tout apiculteur sait en gros ce fu'on trouve dans le miel.

\section{Composition du miel.}

Fin moyenne, le miel contient 20 p. Ioo d'eau, 75 p. Ioo de sucres diver: et 5 p. 100 de constituants mineurs mais d'une extrême variété, dont l'inventaire est bien loin d'être achevé et qui connent au produit sa véritable personnalité. Cela, tout apicultent le sait, on derrait le savoir.

Classiquement on considère que les sucres du miel sont le glucose, le lévulose et le saccharose. Glucose et lévulose proviennent de l'invertion du saccharose contenu dans le nectar par les enzynes (invertases) cle l'abeille : le saccharose du miel est considéré connne un reste ayant échappé à l'invertion.

lin fait, les techniques de la chimie classigne étaient totalement insuffisantes pour pousser plus loin l'identification des sucres du miel. Villes donnent des résultats globaux qui sont certe intéressants mais qui ne rendent pas concompte de l'exacte réalité. Avec l'apparition de la chronatographie sur papier et son application anx sucres du miel, on s'est aperçu dul' ils sont bien loin l'être aussi simples qu'on le croyait. C'est ainsi que Tärfir. et Risss (I0.52) ont trouvé dans le niel cinc sucres totalement inconnus jussu'à ce jour et quil, pour l'instant ne portent pas encore de nom. Par contre, ils ont pu démontrer que le saccharose n'est pas aussi commun dans les miels qu'on le pensait avant eux. Il serait souvent confondu avec d'autres sucres avec lespuels il présente de grandes analogies chiniques. Plus récemment, Wurris et Manzr ont mis en évidence une dizaine de sucres nouveaux présents en petites quantités clans le miel et ils ont montré que les enzynes de l'abeille ne travaillent pas selon le schéma simple admis jusqu'alors : saccharose - glucose $\because$ lérulose. Il y a en effet possibilité de transformation clu glucose en lévulose par isomérisation sous l'influence des eny.mes, si bien que le fameux rapport lévulose iglucose qui devrait être relativement fixe, se montre an contraire essentiellement rariable.

Quant à ce qu'on appelle habituellement la dextrine clu miel et que l'on obtient par précipitation par l'alcool, il est maintenant établi qu'il s'agit d'une fraction du miel particulièrement complexe et qui n'a probalılement pas grandchose à voir avec ce qu'on appelle habituellement dextrine.

l)ans le domaine des acides organiqu's du miel, même progrès. Whitr: (I957) travaille actuellement cette question par la chronatographie sur papier. Il a déjà mis en évidence une quinzaine d'acides organiques dont 4 seulement ont pu être identifiés. Le travail continue.

Pas de grands progrès à propos des citamines. Ise miel est pauvre ou même très pauvre en ce genre de substances. Il est bien établi maintenant que la valeur du niel ne saurait résicler sans sa teneur en vitannines.

L'attention des climistes se porte plutot maintenant vers les diastases et on peut dire qu'il y a une véritable querelle des diastases du niel, querelle qui est bien loin de n'avoir qu'un caractère strictement académique. Il s'agit, en effet, d'une question qui intéresse tout le commerce du miel.

Que sont donc ces famenses diastases? In bref, ce sont des corps très actifs présents à l'état de traces dans la matière vivante; elles permettent à la température ordinaire les réactions climi(pues indispensables à la vie. Cesont donc des catalyseurs biologiques. I, invertase par exemple permet l'hydrolyse d'un strcre complexe, le saccharose en sucres plus simples pouvant être assiniles 
par l'organisme. I'anılase dégrade l'amidon en sucres simples de façon analogue.

Les miels naturels sont, le plus souvent, riches en diastases, tant d'origine animale que d'origine végétale. Par ailleurs, ces diastases sont assez sensibles à la chaleur et elles sont détruites ou affaiblies par une élévation importante de température. On peut donc se servir des diastases comme indicateur de chauffage des miels ; tout niel chauffé exégarément présente des réactions diastasiques affaiblies ou nulles, ce qui permet aux pays importeurs de miel et aui ne tolèrent pas le chanffage, de refouler comme impropres à la consommation les miels n'ayant pas de réaction diastasique suffisanment positive.

Malheureusement, il y a (les miels naturels (miel d'oranger par exemple) uui présentent des réactions diastasiques nulles ou très faibles, mêne sans chauffage. I'où la querelle des diastases entre les pars importateurs qui exigent res miels une réaction diastasique positive comme preuve du non-chanffage et les jays exportateurs qui protestent contre une réglementation qui leur parait abusive.

Que faut-il penser de cette querelle? I)u point de vue diététiune la présence ou l'absence de diastases dans un miel parait assez indifférente. Il y a plus d'amylase dans une goutte de salive que dans quelques centaines de grammes de miel. Quant à l'invertase, elle est normalement sécrétée par nos glandes digestives et les traces d'invertase que contient le miel ne sont sans doute pas d'une bien grande utilité pour notre digestion. Ia plupart des sucres du niel sont d'ailleurs directement assimilables sans digestion et c'est là l'une des principales qualités du produit des abeilles.

I,es diastases constituent donc plutôt un moven, d'ailleurs contesté, d'apprécier la qualité d'un miel qu'une qualité proprement dite. Reste à savoir si un chauffage modéré du micl, tel qu'il apparait nécessaire pour garantir un conditionnement correct, est vraiment nuisible au miel, et à (juels constituants du miel. Nous ne trancheron's pas cette question car tout dépend de ce 'qu'on entend par chauffage. KIERMEIER et KöHERLEIN (I954) qui ont étudié l'inactivation des diastases par la chaleur ont soumis les miels à des chauffage de 2 à + lieures; les méthodes modernes ne font porter le chauffage que sur quelques minutes et nous sommes persuadés que dans ces conditions les dommages causés au miel sont insignifiants. IIn gros travail de recherche reste encore à faire dans ce domaine, surtout en étudiant de très près les propriétés biologiques du miel.

Il resterait encore beaucou1 à dire sur les autres constituants mineurs du miel : sels minéraux, collö̈les, protéines, pignents, substances odorantes, hormones, inhibine, etc... I les centaines de corps restent à identifier dans le miel à l'état de traces; ce sont eux qui font que le miel ne ressemble à rien d'autre.

\section{Problèmes de torhmologio}

Nous voici donc en possession de données qui nous permettent maintenant d'envisager les problèmes de technologie du miel avec de plus grandes chances de les résoudre de façon satisfaisante. Ces problènes sont fort nonbreux et nous ne pouvons pas les envisager tous aujourd'hui. Il convient donc de les classer par grands groupes et d'examiner pour chayue groupe les solutions les plus intéressantes. J'insisterai tout spécialement sur les problèmes qui sont à l'étude à la Station expérimentale d'apiculture de Iontfavet.

l)ans l'ordre chronologifue des opérations se posent en premier lieu des 
problèmes de récolte et de manutention. Comme il n'est plus guère fait usage de la presse pour extraire le miel, c'est nniquement l'extractenr centrifuge (pui retiendra notre attention. Cet appareil semble avoir atteint un état de perfectionnement difficile à dépasser. Pourtant, nous savons tous que le taux d'extraction des appareils actuellement en service, s'il est voisin de Ioo p. Ioo n'atteint pourtant jamais ce chiffre yu'il est d'ailleurs théoriquement impossible d'atteindre. Mais, entre 95 et $(9)$ p. Ioo d'extraction par exemple, la marge est grande. Admettons qu'un perfectionnement apporté à l'extracteur classique, ou simplement à la façon de l'utiliser, permette d'augmenter ne serait-ce que de i p. Ioo le taux d'extraction sans rien changer aux opérations habituelles, le productenr de $\mathrm{I}$ o tonnes de miel aura récolté en fin de saison $100 \mathrm{~kg}$ de miel en plus qui ne lui auront rien coùté. Je suis persuadé qu'une simple étude rationnelle des taux d'extraction en fonction de la température du niel et de sa viscosité ainsi (que de la vitesse de rotation de l'extracteur et du temps d'extraction permettrait d'améliorer le rendement de bien des installations où le temps de fonctionnement de l'extracteur est fixé un peu au hasard.

Ceci n'est 'fu'un point de la technologie de l'extraction. Il en est bien d'autres qui mériteraient une étude approfondie. Il y a notamment dans ce lomaine une quantité très importante de problèmes qui relèvent surtout de l'organisation scientifique du travail. Ine organisation rationnelle de la miellerie c'est-à-dire une disposition juclicieuse des lieux, un calcul serré cles temps d'extraction, une certaine mécanisation des manipulations de hausses par l'emploi des palettes, le pré-chauffage des hausses qui facilite l'extraction du niel, tout cela est de nature à élever sensiblement le rendement du poste d'extraction, donc de réduire le nombre d'heures de travail d'extraction par tonne de miel. Nous avons tenté à IIontfavet de réaliser une installation aussi rationnelle que possible en ce (qui concerne la disposition des lieux et l'emplacement des appareils les uns par rapport aux autres.

'lant que le miel reste à l'état liquide, le moven le plus rationnel d'en assurer la manutention est de le faire circuler par pompage ou par gravité dans des canalisations appropriées. Il est anachronique de voir encore à l'heure actuelle des installations d'extraction de professionnels où le miel est repris au seau à la sortie de l'extracteur pour être versé dans le maturateur. Ines canalisations doivent avoir un dianètre compris entre 3,5 et $i$ cm ; elles peuvent être en plastique, en pyrex (solution récente et qui parait fort intéressante) ou en inox, solution couteuse mais parfaite. Le pompage ne peut se faire çu'au moyen de pompes à mouvements lents et à grandes ouvertures. Il faut proscrire tout système qui incorpore de l'air au miel.

On prévoit généralement à la sortie de l'extracteur un poste de filtration sommaire; des tôles perforées arrêtent les clébris de cire jusıu'à un diamètre de I ou $2 \mathrm{~mm}$. I,e miel est envoyé ensuite au maturateur.

Si nous considérons l'ensemble des opérations allant cle l'extracteur centrifuge à la clarification du miel au maturateur nous constatons que partout nous avons besoin cl'un miel fiuide ou très flude. L'ne bonne fluidité du miel ne peut être acquise que vers $35^{\circ} \pm 5^{\circ}$ selon la teneur en eau. Cette température idéale n'est (que fort rarement réalisée dans la mielle rie. I a température ordiniare des locaux d'extraction ne dépasse guère 20 à $25^{\circ}$, soit un déficit d'une dizaine de degrés environ; sur la viscosité le gain apporté par un écart de $0^{0}$ est considérable ; le miel est rendu 3 à + fois plus fluide. I.e pré-chauffage des hausses peut se faire aisément clans un local doté d'un chauffage électrique réglé par thermostat. Il faut tenir compte de la très mauvaise conductibilité thermique 
du niel et des difficultés de circulation de l'air chaud dans les hausses. Il y a donc lieu de prévoir un passage en chambre de pré-chauffage pouvant atteindre ou dépasser 24 heures. I es controles de température sont indispensables pour déterniner le moment oì la nasse du miel a atteint sa température optina : pour chaque installation l'expérience peut seule faire connaitre les temps de pré-chauffage à applipuer.

Si le local d'extraction n'est pas rraiment froid on pent consiclérer que la perte de chaleur entre désoperculation et sortie de l'extracteur est négligeable. Par contre. dès la filtration sommaire il faut réchauffer le miel et le ramener à $35^{\circ}$ environ. Nous avons prévu pour cela un petit chanffage électrique auxiliaire sous la cuve de filtration. Nous avons ainsi la garantie gue le miel ne sera pas trop visqueux au moment du pompage et qu'il arrivera aux maturateurs sans s'être sensiblement refroidi. Dans les maturatenrs enx-mêmes la remonté cles bulles d'air et des impuretés diverses ne peut se faire rapidement et correctement que si la viscosité (les miels est faible. I)'autre part, il est très important d'entraver toute cristallisation prématurée dans le maturateur, cristallisation yui s'amorce spontanément en quelques jours pour les miels de col\%a par exemple et pour quelques antres miels.

Nous pensons donc qu'il faut prolonger la chaine de chaleur jusqute dans les maturateurs. Nous savons d'ailleurs que le miel ne demande qu'à se maintenir chaud et nous n'avons besoin de prévoir pour les maturateurs qu'un chaufffage d'appoint destiné à compenser les pertes, pourvu bien entendu que l'ensemble soit correctement calorifugé.

Dans notre installation de Montfavet les maturateurs sont calorifugés au moyen de playues de frigolithe et réchauffés au moven de petites résistances commandées par thermostat (jui maintiennent toujours le miel à cette température de $35^{\circ}$, qui est celle de la ruche à I ou 2 clegrés j)rès et (pui ne saurait donc être considérée par les esprits les plus chagrins comme une température inadmissible pour le miel.

A $35^{\circ}$ la clarification est rapicle, la cristallisation ne peut pas se faire et it n'y a pas non plus de fermentation. Seul inconvénient de l'opération, un léger changement de coloration du miel qui peut devenir perceptible au bout de quelques semaines. Cet inconvénient est mineur car nous ne considérons pas le maturateur comme un lieu de stockage mais unicuement comme le lieu ou s'épure le miel par décantation.

Ainsi se trouve donc réalisée la chaine de chaleur (jue nous considérons comme indispensable tout d'abord à une extraction facile et totale, puis à une épuration satisfaisante.

Les problèmes qui se posent désormais sont des problèmes de stockagr et de conditionnement. C'est là qu'interviennent la teneur en eau et la nature du miel pour aiguiller l'apiculteur vers telle ou telle technique de conditionnement. Je crois que l'on peut poser comme principe général qu'un miel qui titre plus de Is' p. Ioo d'humidité est voné à une fermentation plus on moins rapide et plus ou moins importante selon le nombre de germes présents à l'origine et qui peut varier de I à plusieurs millions par gramme. Relativement faibles à Is p. IOO, les risques sont considérables dès qu'on atteint ou dépasse 20 p. 100. I'autre part les miels titrant plus de is p. Ioo d'eau sont sujets à l'effondrement de la texture cristalline, c'est-à-dire à la séparation en deux phases, l'une liquide contenant le lérulose et qui fermente arec la plus grande facilité, l'autre solide constituée surtout de glucose.

Ira rectification de la teneur en eau des miels parait devoir s'imposer dans 
beaucoup de régions. Il m'est passé par les nuains plus de I 200 échantillons de miel au cours de ces dernières années et je peux affirmer que l'on rencontre des miels fermentés ou fermentescibles dans toute la Firance. L'apicultenr n'est d'ailleurs pas toujours responsable de cet état de choses : il est prouvé que le tanx d'humidité pour lequel a lien l'ouerculation est extrêmement variable (de io à plus de 26 p. Ioo) selon les conditions locales.

Jeux solutions peuvent être envisagées :

I $^{\circ}$ Déshydratation du miel avant l'extraction par circulation d'air chaud dans les piles de hausses.

$2^{\circ}$ I éshydratation du miel extrait.

La prenière de ces techniques a été préconisée à plusieurs reprises par les

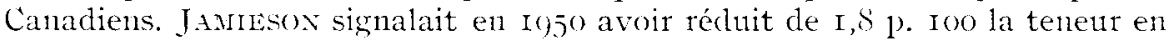
ean du miel contenu dans une pile de hausses par circulation d'air à $40^{\circ}$ et 33 p. IoO d'humidité relative. I' autres essais ont eu lieu dans le même sens et, à chaque fois, le procédé a donné satisfaction. Nous comptons le mettre en application à Iontfavet. I,e dispositif expérimental que nous devons utiliser comportera une section de réchauffage de l'air sur des résistances électriyues. la circulation à l'intérieur cles piles de hausses, enfin une section de déshyclratation de l'air par condensation de la vapeur sur des éléments réfrigérés à très basse température. Le même air est constamment recyclé. L'analyse des eaux de condensation obtenues par dégivrage nous permettra de rechercher les parties volatiles du miel susceptibles d'être enlevées par le procédé. I'avantage du dispositif est de constituer à la fois la chambre de pré-chanffage des hausses à $35^{\circ}$ et la chanbre de déshydratation.

La seconde solution consiste à déshydrater le miel extrait par passage clans un appareil à évaporation sous vide. Les Néo-Zélandais, qui récoltent toujours des miels trop riches en eau pour pouvoir être exportés, ont mis au point un appareil qui leur donne toute satisfaction. Il comporte un cylindre en acier inoxydable à double paroi, chauffé à la vapeur et dans lecuuel on fait un vide de $68 \mathrm{~mm}$ de $\mathrm{Hg}$. Ce cylindre est relié à un conclenseur, où l'eau arrachée au miel se condense, et à une pompe à vide. Le miel humide tombe en pluie dans le cylindre et un ingénieux système de sas à dépression permet d'évacuer régulièrement le miel traité. L'appareil revenait à 150000 Fr environ, il y a quelques années, mais il faut ajouter au prix de l'appareil celui d'un générateur à vapeur et d'une ponpe. I es perfornances sont les suivantes : débit 3 à $4 \mathrm{kgr}$ de miel par minute. Réduction de la teneur en eau : I à 3,7 p. 100 . 'l'empérature du miel : $50^{\circ}$ environ. lin 8 heures de fonctionnement l'applareil retire 2 p. Ioo de son eall à $2500 \mathrm{~kg}$ de miel. Le fonctionnement cesse d'être satisfaisant si la teneur en eau dépasse 20 p. Ioo au départ par suite cl'une insuffisance du condenseur à laquelle il serait d'aitleurs facile cle remédier. La qualité du miel traité est très améliorée.

Nous comptons installer à Montfavet un dispositif de déshyclratation du miel extrait nettement plus simple que celui qui vient d'être décrit. I,a chambre de pré-chanffacre et déshydratation serait directenlent utilisée. I'appareil est encore à l'état de projet. I) toute façon, nous considérons qu'il n'y a pas de problème difficile à résoudre du côté de la déshyclratation des miels. Les principes à appliquer sont connus et le matériel sera rapidement mis au point. Il faudra ensuite chercher à réaliser à un prix aussi bas que possible les installations courantes.

Le problème que nous allons maintenant aborder est celui de la stalilisation de l'itat phy'sique des miels. C'est un problène de présentation et de con- 
ditionnement. L'enquête (que nous avons faite l'an dernier sur la vente du miel à Paris nous a prouvé qu'il s'agissait d'une question grave pour l'avenir de la consommation du miel. La qualité moyenne des miels offerts dans le commerce ne peut être sensiblement améliorée que si nous adoptons des techniques modernes de stabilisation. lìt là, deux possibilités s'offrent à nous : miel liquide ou miel solide. Selon que l'on choisit l'une ou l'autre solution les techniques à appliquer sont totalement différentes.

Le miel vendu à l'état solide cloit présenter les qualités suivantes : propreté, stabilité de la cristallisation, résistance à la fermentation, granulation très fine et très homogène. La propreté doit pouvoir être obtenue par le simple passage au maturateur. I a filtration poussée ne parait pas indispensable. Ia stabilité de la cristallisation, c'est-à-dire la garantie que le miel ne se séparera pas spontanément en ses constituants solides et liquides, ne peut être acquise (que par un examen sérieux de la composition du miel. Si on se base sur les traval1x de JAmIESON, la stabilité de la cristallisation est fonction de la teneur en eau et clu rapport lérulose/glucose. (n peut résumer comme suit les résultats de Jamieson : pour in p. Ioo d'humidité, le miel est stable quel que soit

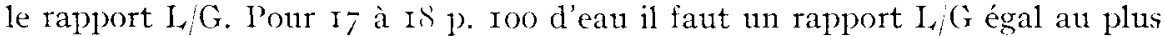
à I,I4. Pour Io) p. IoO d'eau il faut un rapport $\mathrm{I} / \mathrm{G}$ égal à 1,0 . Pour 20 p. 100 d'eau et au-delà il 11'y a plus de stabilité de texture assurée.

İ̀n conclusion, les miels ayant vocation pour être présentés à l'état solide sont des miels pauvres ou très panvres en eau et riches en glucose. La faible teneur en eau est favorable à une bonne conservation. I a pasteurisation ne s’impose pas du moment cu'on ne dépasse pas 17,5 à Is p. Ioo d'humidité. Pour obtenir la granulation très fine qui seule permet de vendre des miels agréables malgré leur cristallisation on appliquera bien entendu le procédé qui consiste à mélanger au miel à $25^{\circ}$ une semence très fine, à remplir les pots de détail avec la masse pâteuse et à faire cristalliser en chambre fraîche à $4_{4}^{\circ}$.

l,e miel vendu à l'état liquicle doit présenter les propriétés suivantes : propreté très poussée, stabilité de l'état liquicle pour au moins six mois, résistance à la fermentation. I a propreté très poussée pent être obtenue, soit au maturateur, soit par filtration a chaud. La stabilité de l'état liculuide et la résistance à la fermentation ne peuvent être obtenues que par la pasteurisation. Malgré les avantages de la pasteurisation et les garanties qu'elle offre on choisira de préférence pour la présentation à l'état lípuicle des miels riches en lévulose (rapport I/G '́levé). Il ne faut pas oublier que pour une teneur en eau identique de $I 7$ p. Ioo la vitesse de cristallisation spontanée à I $^{\circ}$ va de $u n$ mois pour un rapport $\mathrm{I}_{\mathcal{A}} \mathrm{G}=\mathrm{I}, 00$, à 27 mois pour un rapport $\mathrm{I} / \mathrm{G}=\mathrm{G}, 5.5$.

I,e but de la pasteurisation est double : I $^{0}$ tuer tous les germes susceptibles ('entraîner la fermentation dı miel ; $2^{\circ}$ détruire tous les cristaux primaires de glucose qui sont à l'origine de la prise en masse du miel.

On sait yu'un chauffage de $30 \mathrm{~mm}$ à $62^{\circ}$ tue toutes les levures. Un chauffage de $5 \mathrm{~m} n$ à $\overline{7}^{\circ}$ a le même effet. Comme le tenus de chauffage doit être aussi court que possible la seconde solution est préférable.

I a destruction des cristaux primaires pose clavantage de problèmes. Sans entrer dans le détail des travaux américains sur cette question, signalons que la pasteurisation doit être d'autant plus longue et la température d'autant plus élevée que la cristallisation à clétruire est plus grossière. Pratiquement il faut considérer (qu'une pasteurisation vraiment rapide n'est eflicace (que sur un miel préchauffé et (qui arrive déjà très fluide à l'applareil. Le pasteurisateur n'est pas destiné à fondre le miel mais à changer de façon permanente son état physique. 
I.e bac de préchauffage s'impose. l)ans notre installation il sera constitué simplement par le maturateur chauffé.

I, e pasteurisateur A. I'. V. dont nous avons fait l'acquisition est avant tout t11 appareil expérimental. Il comporte des perfectionnements qui n'auront pas leur place sur un appareil courant travaillant, non pas à la demande, mais dans des conditions rigoureusement fixées à l'avance.

Schématiquement, voici comment fonctionne notre apareil. I,e miel liquide porté à $35^{\circ}$ environ, donc pratiquement sortant du maturateur, est injecté sous pression par une pompe entre des plaques d'acier inoxydable poli enupilées comme des assiettes et ne laissant entre elles qu'un espace cle quelques mm. De l'eau chaude (température réglalıle ejtre bo et $00^{\circ}$ ) circule également entre les pląues dans un circuit distinct et communique sa chaleur au miel en un temps très court. Cette eau chaucle est obtenue au moven d'un génératenr de vapeur haute pression et d'un injecteur. I'injecteur est contrôlé de façon très rigoureuse par un dispositif thermostatique très précis, si hien que la température de pasteurisation fixée au clépart ne varie pas de plus d'un degré. Un thermomètre enregistreur permet de suivre à tout instant les éarts de température éventuels, ce qui est très important dans une installation expérimentale.

Iès y j'il est amené à la température voulue (en principe $\left.\tau^{\circ}\right)^{\circ}$ le miel passe dans un circuit de clambrage (jui permet de maintenir le produit à cette température pendant le temps nécessaire. Notre appareil doit comporter un jeu de tubes de pyrex permettant de réaliser des temps (le chambrage variables. I)ans une autre version qui est à l'étude le circuit de chambrage sera constitué par 111 filtre à mailles très fines capable d'arrêter les impuretés jusqu'à $\mathrm{I} / \mathrm{IO}$ de mm.

A la sortie du circuit de chambrage le miel retourne dans le pasteurisateur et passe dans un nouveau jeu de plaques qui sont réfrigérées par de l'eau à $20^{\circ}$. A la sortie de l'appareil le miel est tiède et pent être directement mis en pots. Le débit de l'appareil est de $250 \mathrm{kgh}$, tout au moins pour le moment : des possibilités de développement sont prévues atin d'ajuster exactement le débit du pasteurisateur à celui d'une machine à empoter.

Ionc, rien de comparable entre la pasteurisation-éclair où tout est minutieusement contrôlé et un chauflage sans précaution, irrégulier, suivi d'un refroidissement lent qui maintient pendant des henres on même des jours entiers le miel chauct au contact de l'air et de métaux plus ou moins oxyclables. I.aspect du miel pasteurisé n'a pas changé ; la coloration est la mème : l'élimination des derniers cristaux lui donne nue linupidité qu'il doit pouvoir garder pendant atl moins six mois.

J'en terminerai maintenant en donnant le schéma idéal d'une installation de conditionnement de miel, telle que nous la concevons actuellement. Nous trouvons au début de la chaine, la chambre de pré-chauffage des hausses et de déshydratation avant extraction. I'uis, vient le poste d'extraction comportant la désoperculation. An sortir de l'extracteur et de la cuve à désoperculer le miel est sommairement filtré, amené à $35^{\circ}$, pompé et envoyé au maturateur à température constante où s'achève l'épuration. Dans un premier type de cycle de conditionnement le miel est pasteurisé, mis en pots et conservé au froid $\left(+4^{\circ}\right)$ jusqu'à la vente. l)ans un second type de cycle il est envoyé au mélangeur de semence, nis en pots et conservé à i $f^{\circ}$ température idéale pour la cristallisation. On peut considérer encore plusieurs variantes, avec retour an déshydrateur, ou bien pasteurisation avant ensemencement (procédé Dyce classique), ou bien enfin chaine continue de lextracteur à la mise en pot dans la même journée la clarification étant remplacée par une excellente filtration. 
Nous nous efforçons de réaliser progressivement la miellerie-type, qui d'ailleurs dlans notre esprit ne pent être r un'une niellerie coopérative de façon à assurer $11 n$ fonctionnement rentable à cles appareils clont certains sont assez coûteux. Cette réalisation ne peut pas se faire d'un seul coup car les crédits d'équipentent qui nous sont accordés ne nous arrivent pas en bloc. mais par fractions annuelles ne permettant à chaque fois que la réalisation d'une partie clu programme. Nous pensons ([ue l'année 1954 ) verra la mise en place de tont le gros matériel actuellement prévu. L'été I gro devrait voir l'achèvement de nos projet; actuels.

\section{RFIITIECES BIBIIOCRAPHIOLES}

Helver ('l. C.). - Study on some plissical properties of honey. Fond Research, 19 (3), 2\$2-292, I954.

JAmison (C. A.). - In " Bee I ivision, Central experimental farm Ottawa Progress report $1\left(937-\mathrm{I} 048\right.$ " Ottawa $195^{\circ}, 47$ pages.

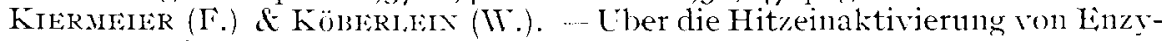
men in Honig. $Z$. Le lensmitt. ('nters., 98 (5),32(3)-347, r954.

Muno (J.A.). - The riscosity and thixotropy of honey. J. econ. Ent., 36, 7og$777, \quad I 943$.

PRYce-Joxes (J.). - - T'he rheology of honey. In "Foodstuffs, their plasticity, fluidity and consistency \%. Amsterdam 1953.

Täris (K.) \& REISs (R.). - Analytische und chromatographische Studien an Bienenhonig. Z. Lebensmit. Lnters., 94 (I), I-IO, I952.

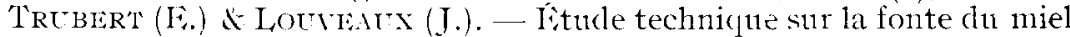
cristallisé. Ann. Abille, I (I), I()-30, I958.

Whirte (J. W. jr.) — The composition of honey. Bee Trorld, 38 (3), 57-66, I 957 . 